\section{Abdominal aortic calcification predicts mortality in hemodialysis patients}

Abdominal aortic calcification, which can be detected using a standard radiograph of the lateral abdomen, is a predictor of cardiovascular mortality in the general population. Researchers have investigated whether abdominal aortic calcification also predicts mortality in hemodialysis patients, who often have vascular calcification.

The study included 515 patients on maintenance hemodialysis at a hospital in Japan. All patients underwent radiography of the left abdomen at baseline.

Abdominal aortic calcification was present in 291 (56.5\%) patients. During a mean \pm SD follow-up period of $51 \pm 17$ months, 103 patients died; 81 of these were patients with abdominal aortic calcification at baseline, and 22 were patients without such calcification. More than one-third of deaths were caused by cardiovascular conditions such as ischemic heart disease and congestive heart failure. Patients with abdominal aortic calcification were significantly more likely to die from any cause $(P<0.0001)$ or from a cardiovascular cause $(P=0.0001)$ than were patients without abdominal aortic calcification. The presence of abdominal aortic calcification was also found to be associated with all-cause mortality (hazard ratio 2.07) and cardiovascular mortality (hazard ratio 2.39) in multivariate analyses adjusted for factors such as age, presence of diabetes, creatinine level and C-reactive protein level.

The authors conclude that abdominal aortic calcification, which can be screened for easily during routine visits, might be a useful prognostic indicator in hemodialysis patients.

Original article Okuno S et al. (2007) Presence of abdominal aortic calcification is significantly associated with all-cause and cardiovascular mortality in maintenance hemodialysis patients. Am J Kidney Dis 49: 417-425

\section{Risk of renal failure after bariatric surgery in patients with kidney stones}

Jejunoileal bypass was the first widely applied form of surgical treatment for obesity. Hepatic and renal complications-including nephrolithiasis secondary to hyperoxalurialed to this procedure being banned in the US in 1980. Asplin and Coe have evaluated the prevalence of hyperoxaluria in a group of obese kidney stone formers who had undergone currently used bariatric procedures such as gastric banding and resection.

The authors analyzed urine chemistry data of 132 patients with nephrolithiasis who had undergone bariatric procedures and compared them with data from 27 patients who had undergone jejunoileal bypass, 2,048 nonsurgical patients with kidney stones, and 168 healthy individuals.

Rates of urinary oxalate excretion in patients who had undergone bariatric surgery were higher than those in untreated kidney stone formers or healthy individuals (mean values of $83 \mathrm{mg}$ per day vs $39 \mathrm{mg}$ per day and $34 \mathrm{mg}$ per day, respectively; $P<0.001$ for both comparisons), but less than those of patients treated with jejunoileal bypass (102 mg per day; $P<0.001)$. Supersaturation of urine with calcium oxalate-the cause of calcium oxalate stone formation-was highest in patients who had undergone bariatric surgery $(12.1 \pm 0.5$ in bariatric surgery group vs $9.0 \pm 0.1$ in untreated stone formers, $7.4 \pm 0.3$ in healthy individuals, and $8.9 \pm 1.1$ in those who had undergone jejunoileal surgery).

Clinicians should be aware of the possibility of patients developing stone disease and renal damage after bariatric surgery.

Original article Asplin JR and Coe FL (2007) Hyperoxaluria in kidney stone formers treated with modern bariatric surgery. J Urol 177: 565-569

\section{Use of a timesheet cuts cold ischemia time of donor kidneys by 8 hours}

In renal transplantation, prolonged cold ischemia time (CIT) is associated with delayed graft function and poor long-term outcome. Researchers from Marseille, France, report the impact that recording the timings of interventions had on $\mathrm{CIT}$ at their transplant center. Timesheet keeping was initiated in June 2004; all timesheets up to April 2006 (121 transplants) were retrospectively analyzed, and results compared with those of the preceding 2-year period (151 transplants; no timesheets). 